\title{
RIESZ TRANSFORMS ON SPHERES
}

\author{
Nicola ArcozZi And Xinwei Li
}

\section{Introduction}

This note explores, on the sphere $\mathbb{S}^{n-1}=\left\{x \in \mathbb{R}^{n}:|x|=1\right\}$, several analogues of the classical Riesz transforms on $\mathbb{R}^{n}$. Recall that, if $f \in L^{1}\left(\mathbb{R}^{n}\right)$, then

$$
R f(x)=p \cdot v \cdot c_{n} \int_{\mathbb{R}^{n}} f(x-y) \frac{y}{|y|^{n+1}} d y
$$

is the vector valued Riesz transform of $f . c_{n}$ is a constant that only depends on the dimension and that is chosen so that $\left(R_{j} f\right)^{\wedge}(\xi)=i \frac{\xi_{j}}{|\xi|} \hat{f}(\xi)$, where $R_{j} f$ is the $j$-th component of $R f, \xi=\left(\xi_{1}, \ldots, \xi_{n}\right)$, and $\hat{f}$ is the Fourier transform of $f$.

It is well known that there are several equivalent ways to define $R$. One way is related to a Neumann problem. Let $F$ be the solution in the upper half space $\mathbb{R}_{+}^{n+1}=\mathbb{R}^{n} \times[0, \infty)$ of

$$
\Delta_{\mathbb{R}^{n+1}} F=0 \quad \text { in } \mathbb{R}_{+}^{n+1}, \quad \frac{\partial F}{\partial \nu}=f \quad \text { in } \mathbb{R}^{n},
$$

where $f$ is a Schwartz function, $F(x, t)$ is bounded for $t \geq t_{0}>0, \forall t_{0}>0$, and $\frac{\partial}{\partial \nu}$ is the outward normal derivative to $\mathbb{R}_{+}^{n+1}$ at the boudary $\partial \mathbb{R}_{+}^{n+1}=\mathbb{R}^{n}$. Then, $R f=\nabla_{\mathbb{R}^{n}}\left(\left.F\right|_{\mathbb{R}^{n}}\right)$ or, formally,

$$
R f=\nabla_{\mathbb{R}^{n}} \circ\left(\frac{\partial}{\partial \nu}\right)^{-1} f,
$$

where $\left(\frac{\partial}{\partial \nu}\right)^{-1} f$ means the restriction to $\mathbb{R}^{n}$ of the solution $F$ of (0.2). An easy argument (for example, via Fourier transforms) shows that $\left(-\Delta_{\mathbb{R}^{n}}\right)^{\frac{1}{2}}=\frac{\partial}{\partial \nu}$. Thus, formally, $R=\nabla_{\mathbb{R}^{n}} \circ\left(-\Delta_{\mathbb{R}^{n}}\right)^{-\frac{1}{2}}$ (see, for example, Stein [St1]).

We can naturally identify $\mathbb{S}^{n-1}$ as the boundary of two Riemannian manifolds. On the one hand, $\mathbb{S}^{n-1}=\partial \overline{\mathbb{B}}^{n}$, where $\mathbb{B}^{n}$ is the open unit ball in $\mathbb{R}^{n}$. On the other hand, $\mathbb{S}^{n-1}$ is the boundary of the cylinder $\mathbb{S}^{n-1} \times[0, \infty)$. By replacing $\nabla_{\mathbb{R}^{n}}$ in $(0.3)$ with the spherical gradient $\nabla_{\mathbb{S}^{n-1}}$, one obtains two nonequivalent definitions of Riesz transforms on $\mathbb{S}^{n-1}$, that we call of ball type and cylinder type (see $R^{b}$ and $R^{c}$ in $\S 1$ ).

Received July 21, 1995, Revised March 12, 1997.

1991 Mathematics Subject Classification: 42B20.

The first author was partly supported by a grant from the Istituto Nazionale d'Alta Matematica (INDAM). 
The Riesz transform on $\mathbb{R}^{n}$ can also be characterized in terms of invariance properties with respect to dilations and translations. This suggests yet a third definition on $\mathbb{S}^{n-1}$, where the invariance is in relation to some unitary representations of $S O(n)$. See $\S 1$.

In the three transforms on $\mathbb{S}^{n-1}$, it is natural to replace $\nabla_{\mathbb{S}^{n-1}}$ with left invariant vector fields in the Lie algebra of $S O(n)$. This leads to a new version for each Riesz transform on the sphere.

Another equivalent way to define Riesz transforms on $\mathbb{R}^{n}$ is by means of Riesz systems, or systems of conjugate harmonic functions, on $\mathbb{R}_{+}^{n+1}$ (see [St2] and $[\mathrm{SW}]$ ). Riesz systems can be defined on the unit ball $\mathbb{B}^{n}$ as well, giving rise to operators that do not belong to our family of Riesz transforms on the unit sphere.

In $\S 1$, we give the three definitions, each in two versions. In $\S 2$, we discuss some results about their $L^{p}$ norms, for $1<p<\infty$, some of which are new. In $\S 3$, we relate Riesz transforms on $\mathbb{S}^{n-1}$ and $S O(n)$, with applications to their $L^{p}$ estimates. In $\S 4$, we summarize some known results about Riesz systems on $\mathbb{S}^{n-1}$ and we announce some better $L^{p}$ estimates and a weak $L^{1}$ estimate that seems to be new.

Most operators survived in this note already appear in the literature, but the constants we announce in the corresponding $L^{p}$ estimates improve on the ones previously known. Their proofs will appear elsewhere ([A1] and [A2]). Several problems concerning the Riesz transforms on $\mathbb{S}^{n-1}$ remain open, as can be assumed by the results that we present.

\section{Different Riesz transforms on $\mathbb{S}^{n-1}$ and their $L^{2}$ norms}

As already mentioned in the introduction, there are at least two natural ways to "fill in" $\mathbb{S}^{n-1}$ so that it is the boundary of an $n$-dimensional Riemannain manifold. The first is to consider

$$
M=\overline{\mathbb{B}}^{n}=\left\{x \in \mathbb{R}^{n}:|x| \leq 1\right\}, \quad \text { with } \mathbb{S}^{n-1}=\partial \mathbb{B}^{n}=\partial M .
$$

The second way is to define

$$
M=\mathbb{S}^{n-1} \times[0, \infty), \quad \text { with } \mathbb{S}^{n-1} \equiv \mathbb{S}^{n-1} \times\{0\}=\partial M,
$$

endowed with the product Riemannian metric.

The manifold in (1.2) was considered with more general spaces instead of $\mathbb{S}^{n-1}$ by many authors, for example, P.A.Meyer [Me], Stein [St1], Bakry [Ba], Coifman and Weiss [CW1].

We have, as well, two different vectors of first order differential operators associated with $\mathbb{S}^{n-1}$. The first is, simply, the spherical gradient:

$$
D f=\nabla_{\mathbb{S}^{n-1}} f,
$$


defined as the gradient on $\mathbb{S}^{n-1}$, when this is considered as a Riemannian submanifold of $\mathbb{R}^{n}$. This was the choice of Koranyi and Vagi [KV1] and [KV2] (see also Ricci and Weiss $[$ RW] $)$.

We now discuss the second operator. Let $x=\left(x_{1}, \ldots, x_{n}\right) \in \mathbb{R}^{n}$, and for each $j<k$ consider :

$$
T_{j k}=x_{j} \frac{\partial}{\partial x_{k}}-x_{k} \frac{\partial}{\partial x_{j}}=\frac{\partial}{\partial x_{\theta_{j k}}} .
$$

where $\theta_{j k}=\operatorname{arctg} \frac{x_{k}}{x_{j}}$ is the angular coordinate in the $\left(x_{j}, x_{k}\right)$ plane. $T_{j k}$ can also be thought of as a Casimir operator in the Lie algebra of the orthogonal group, $S O(n)$.

The link between these operators and the geometry of the sphere can be expressed in several ways. For instance,

$$
\sum_{j<k} T_{j k} T_{j k}=\Delta_{\mathbb{S}^{n-1}}
$$

See Bakry [Ba].

We can then consider

$$
D f=\left(T_{j k} f\right)_{1 \leq j<k \leq n}
$$

which is a vector in $\mathbb{R}^{n(n-1) / 2}$. Denote $T=\left(T_{j k}\right)_{1 \leq j<k \leq n}$. From (1.6) and the fact that the operators $T_{j k}$ are self adjoint, it follows that

$$
|T f|=\left|\nabla_{\mathbb{S}^{n-1}} f\right| .
$$

Now, let $M$ be as in (1.1) or (1.2) and $f$ in $C^{1}\left(\mathbb{S}^{n-1}\right)$ with $\int_{\mathbb{S}^{n-1}} f d \sigma=0, d \sigma$ being the Hausdorff measure on $\mathbb{S}^{n-1}$. Consider the Neumann problem

$$
\Delta_{M} F=0 \text { and }\left.\quad \frac{\partial F}{\partial \nu}\right|_{\partial M}=f
$$

where $F$ tends to zero as $t \rightarrow \infty$ when $M=\mathbb{S}^{n-1} \times[0, \infty), \nu$ is the outward normal vector and $\Delta_{M}$ is the Laplace-Beltrami operator on $M$.

In the sequel we use the convention that, if $X$ denotes a space of integrable functions on $\mathbb{S}^{n-1}$, then $X_{0}=\left\{f \in X: \int_{\mathbb{S}^{n-1}} f d \sigma=0\right\}$ is the subspace of the functions in $X$ having null mean.

Definition. We say that a Riesz transform of gradient type on $\mathbb{S}^{n-1}$ is an operator of the form $R f=\left.\nabla_{\mathbb{S}^{n-1}} F\right|_{\mathbb{S}^{n-1}}$, where $F$ is the solution of (1.7). More specifically, we have:

(i) $R^{c}=\nabla_{\mathbb{S}^{n-1}} \circ\left(-\Delta_{\mathbb{S}^{n-1}}\right)^{-\frac{1}{2}}$, is a Riesz transform of cylinder type and gradient type. Here, $M=\mathbb{S}^{n-1} \times[0, \infty)$ (see Stein [St1]);

(ii) $R^{b}=\nabla_{\mathbb{S}^{n-1}} \circ\left(\frac{\partial}{\partial r}\right)^{-1}$, is a Riesz transform of ball type and gradient type. Here $M=D^{n}$ and $\frac{\partial}{\partial r}$ is the derivative in radial direction (see Koranyi-Vagi [KV1]). 
If we replace $\nabla_{\mathbb{S}^{n-1}}$ by $T$ in (i) (resp., (ii)), we obtain the Riesz transform of cylinder (resp., ball) type and rotational type $Q^{c}=T \circ\left(-\Delta_{\mathbb{S}^{n-1}}\right)^{-\frac{1}{2}}$ (resp., $\left.Q^{b}=T\left(\frac{\partial}{\partial r}\right)^{-1}\right)$.

Notice that $Q^{c}$ in (iii) is defined componentwise by $Q_{j k}^{c}=T_{j k} \circ\left(-\Delta_{\mathbb{S}^{n-1}}\right)^{-\frac{1}{2}}$, $1 \leq j<k \leq n$, where $Q^{c}=\left(Q_{j k}^{c}\right)_{1 \leq j<k \leq n}$. $Q^{b}$ is defined similarly.

Next, we consider two other transforms. Let $\omega_{n-1}$ be the volume of $\mathbb{S}^{n-1}$. Let $R_{t}$ and $R_{s}$ be the principal value integral operators on $\mathbb{S}^{n-1}$ having kernels $k^{t}(x, y)$ and $k^{s}(x, y)$, respectively, where

$$
k^{t}(x, y)=\left(k_{j k}^{t}(x, y)\right)_{1 \leq j<k \leq n}, \quad \text { with } \quad k_{j k}^{t}(x, y)=\frac{1}{\omega_{n-1}} \frac{x_{k} y_{j}-x_{j} y_{k}}{|x-y|^{n}},
$$

and

$$
k^{s}(x, y)=\frac{1}{\omega_{n-1}} \frac{y-(x \cdot y) x}{|x-y|^{n}}, \quad x, y \in \mathbb{S}^{n-1} .
$$

These operators are generalizations of the Hilbert transform on the circle, with a good behavior with respect to the action of $S O(n)$ (see (1.8) below). First, observe that, since there is a bijection between all upper triangular $n \times n$ matrices and all skew-symmetric $n \times n$ matrices, we may identify $k^{t}(x, y)$ with a skew-symmetric matrix for $x, y \in \mathbb{S}^{n-1}$. Let $u \in S O(n)$ and $\sigma$ be a skewsymmetric $n \times n$ matrix. Then the mapping $u \rightarrow \tau_{u}^{t}$ defined by $\tau_{u}^{t} \sigma=u \sigma u^{*}$ induces a unitary representation of $S O(n)$ acting on the Hilbert space of the skew-symmetric $n \times n$ matrices with the Hilbert-Schmidt norm (see [CW2]). One can easily show that

$$
k^{t}(u x, u y)=\tau_{u}^{t} k^{t}(x, y), \quad x, y \in \mathbb{S}^{n-1}, u \in S O(n) .
$$

By choosing the unitary representation $u \rightarrow\left(\tau_{u}^{s}\right)=u, u \in S O(n)$, we obtain an expression similar to (1.8) for $k^{s}(x, y)$ and $\left(\tau_{u}^{s}\right)$ as well. Moreover, $R_{s}$ itself commutes with the action $\tau_{u}^{s}$.

Now suppose $n \geq 3$. Let $G(x, y)$ be the Green's function of $\mathbb{R}^{n}$ at $y$; that is

$$
G(x, y)=\frac{1}{(2-n) \omega_{n-1}}|x-y|^{2-n},
$$

where $\omega_{n-1}$ is the Hausdorff measure of $\mathbb{S}^{n-1}$. Let $\tilde{G}$ be the operator defined by

$$
\tilde{G} f(x)=\int_{\mathbb{S}^{n-1}} G(x, y) f(y) d y, \quad x \in \mathbb{R}^{n} .
$$

Then we can reformulate $R_{s}$ and $R_{t}$ as follows:

(iii) $R_{s}=\nabla_{\mathbb{S}^{n-1}} \circ \tilde{G}$, is a Riesz transform of invariant type and gradient type (see $[\mathrm{KV} 1],[\mathrm{CW} 2]$, and $[\mathrm{Li}]$ ). 
Similarly, $R_{t}=T \circ \tilde{G}$ is the Riesz transform of invariant type and rotational type.

We note that the operator $R^{b}$ of Korányi and Vági can be written as the sum of two parts. The first part is just $R_{s}$ and the second part is a sort of average of the first part.

We will see that the six operators just defined are related to each other in several ways. First, we make a simple observation.

Remark 1. When acting on a function $f$, the images of the operators $Q^{b}$ and $R^{b}$ are vectors of different dimensions, but $\left|Q^{b}\right|=\left|R^{b}\right|$. Similarly, $\left|Q^{c}\right|=\left|R^{c}\right|$ and $\left|R_{t}\right|=\left|R_{s}\right|$.

As one would expect, the definitions of the Riesz transforms (i)-(iii) and their rotational counterparts coincide in the case $n=2$. In fact, an inspection of multipliers and kernels implies the following.

Proposition 1. On $\mathbb{S}^{1}$, the operators $R^{c}, R^{b}, Q^{c}, Q^{b}, R_{t}$ and $R_{s}$ coincide with the conjugate function operator $H$ - the Hilbert transform on the cirle.

The following results about the $L^{2}$ norms are useful when studying the size properties of the transforms (i)-(iii). The proofs are easy, and they can be found in [St1], [KV2], [Bak], [A1] and [Li].

Proposition 2. With the same notation as above, we have

(a) $R^{c}$ is an isometric imbedding of $L_{0}^{2}\left(\mathbb{S}^{n-1}\right)$ into $L^{2}\left(\mathbb{S}^{n-1}, \mathbb{R}^{n}\right)$ and $Q^{c}$ is an isometric imbedding of $L_{0}^{2}\left(\mathbb{S}^{n-1}\right)$ into $L^{2}\left(\mathbb{S}^{n-1}, \mathbb{R}^{\frac{n(n-1)}{2}}\right)$;

(b) $R^{b}$ maps $L_{0}^{2}\left(\mathbb{S}^{n-1}\right)$ into $L^{2}\left(\mathbb{S}^{n-1}, \mathbb{R}^{n}\right)$ and $Q^{b}$ maps $L_{0}^{2}\left(\mathbb{S}^{n-1}\right)$ into $L^{2}\left(\mathbb{S}^{n-1}, \mathbb{R}^{\frac{n(n-1)}{2}}\right)$. Moreover,

$$
\left\|\left|Q^{b}\right|\right\|_{2,2}=\left\|\left|R^{b}\right|\right\|_{2,2}=\sqrt{n-1} ;
$$

(c) $\left\|Q_{l m}^{c}\right\|_{2,2}=\left\|Q_{l m}^{b}\right\|_{2,2}=1$;

(d) $R_{s}$ maps $L_{0}^{2}\left(\mathbb{S}^{n-1}\right)$ into $L^{2}\left(\mathbb{S}^{n-1}, \mathbb{R}^{n}\right)$ and $R_{t}$ maps $L_{0}^{2}\left(\mathbb{S}^{n-1}\right)$ into $L^{2}\left(\mathbb{S}^{n-1}, \mathbb{R}^{\frac{n(n-1)}{2}}\right)$. Moreover,

$$
\left\|R_{s}\right\|_{2,2}=\left\|R_{t}\right\|_{2,2}=\left\{\begin{array}{cc}
\frac{1}{2} & , n=3 \\
\frac{(n-1)^{1 / 2}}{n(n-2)} & , \quad n \geq 4 .
\end{array}\right.
$$

Notice that the constant for $R_{t}$ or $R_{s}$ is bounded by $1 / 2$ for all $n \geq 3$.

$$
\text { 2. } L^{p} \text { - norms }(1<p<\infty)
$$

All the operators we called "spherical Riesz transforms" are bounded as operators from $L_{0}^{p}\left(\mathbb{S}^{n-1}\right)$ to itself. This fact is well known; hence, the main boundedness questions concern the best or, at least, "reasonable" constants for these $L^{p}$ inequalities. We present below the best known constants for some of these estimates. The proofs and some more estimates can be found in [A1] and [Li]. 
The techniques in [A1] are probabilistic and we do not discuss them here. They are modelled on methods of Bañuelos and Wang [BW] and Burkholder [Bu], who worked with Riesz transforms on $\mathbb{R}^{n}$. All of the functions considered in this section are real valued. We will see in $\S 3$ how the $L^{p}$ estimates involving $Q^{c}$ can be deduced from analogous estimates involving Riesz transforms on $S O(n)$.

Let $p^{*}$ be the maximum of $p$ and $q$, where $q$ is the conjugate exponent of $p$, $1<p<\infty$. Let $C_{p}$ be the best constant in the $L^{p}$ inequality for the classical Hilbert transform $H$ on $\mathbb{R}$ (see $[\mathrm{Pic}]$ ). Then $C_{p}=\cot \frac{\pi}{2 p^{*}}$. Let $E_{p}$ be the best constant in the $L^{p}$ inequality for the operator $f \rightarrow\left[f^{2}+|H f|^{2}\right]^{\frac{1}{2}}$, which was identified by Essén $[\mathrm{Es}]$ as $E_{p}=\left[1+C_{p}^{2}\right]^{\frac{1}{2}}$.

Theorem 1. [A1] If $f \in L_{0}^{p}\left(\mathbb{S}^{n-1}\right)$, then the following inequalities hold:

$$
\begin{gathered}
\frac{1}{2\left(p^{*}-1\right)}\|f\|_{p} \leq\left\|Q^{c} f\right\|_{p} \leq 2\left(p^{*}-1\right)\|f\|_{p} \\
\frac{1}{\sqrt{n-1}\left(p^{*}-1\right)}\|f\|_{p} \leq\left\|Q^{b} f\right\|_{p} \leq \sqrt{n-1}\left(p^{*}-1\right)\|f\|_{p} \\
\left\|Q^{c}{ }_{l m} f\right\|_{p} \leq C_{p}\|f\|_{p} \\
\left\|Q^{b}{ }_{l m} f\right\|_{p} \leq C_{p}\|f\|_{p} ; \\
\left\|\left[f^{2}+\left|Q^{c}{ }_{l m} f\right|^{2}\right]^{\frac{1}{2}}\right\|_{p} \leq E_{p}\|f\|_{p}
\end{gathered}
$$

and

$$
\left\|\left[f^{2}+\left|Q^{b}{ }_{l m} f\right|^{2}\right]^{\frac{1}{2}}\right\|_{p} \leq E_{p}\|f\|_{p} .
$$

Moreover, the constants in (2.3), (2.4), (2.5) and (2.6) are best possible.

Some remarks are in order. First of all, the constant in (2.1) cannot be the best one, as we see from Proposition 2 in $\S 1$. The constant in the right hand side of (2.2) gives the right value for $p=2$. We also remark that the constants in (2.1) and in the second inequality in (2.2) have the expected rate of growth with respect to both $p$ and $n$.

The first inequality in (2.2) does not exhibit the right asymptotic in $n$. In fact, an analysis of the case $p=2$ on the lines of $\S 2$ shows that for all $f \in L_{0}^{2}\left(\mathbb{S}^{n-1}\right)$ we have that $\|f\|_{2} \leq\left\|Q^{b} f\right\|_{2}$, and that this estimate is best possible.

A consequence of (2.1) and of the fact that (2.3) is best possible is that $\frac{2}{\pi} \leq \lim _{p \rightarrow \infty} \frac{A_{p}}{p} \leq 2$, and $\frac{2}{\pi} \leq \lim _{p \rightarrow 1} A_{p}(p-1) \leq 2$.

The first proof of (2.1) with a constant independent of the dimension $n$ is in $[\mathrm{Bak}]$, where a probabilistic Littlewood-Paley theory is used. So far, no one has given a direct analytic proof of $L^{p}$ estimates for $Q^{c}$ which produces a bound independent of the dimension $n$. The constant in (2.1) is the same as that 
obtained for the vector Riesz transform on $\mathbb{R}^{n}$ by Bañuelos and Wang [BW]. The first claim of a dimension free $L^{p}$ estimate for the vector valued Riesz transform in the Euclidean case was given by E. M. Stein [St3]. But the proof of this result was implied in his earlier books, [St1] and [St2]. Later proofs, with increasingly better constants, were given by Duoandikoetxea and Rubio de Francia [DF], Bañuelos [Bañ], Pisier [Pi], Bañuelos and Wang [BW], Iwaniec and Martin [IM]. In the case discussed in [IM] the arguments apply only to the range $2 \leq p<\infty$. In this range their results are the best known.

Observe that the $L^{p}$ constants in inequalities involving just one of the components of $Q^{c}$ or $Q^{b}$ are the same as one finds in the corresponding inequalities for the Hilbert transform. This phenomenon occurs also for each component of the Euclidean Riesz transform in $\mathbb{R}^{n}$ ([IM] and $\left.[\mathrm{BW}]\right)$. Bañuelos and Wang discovered deep martingale inequalities which seem to explain "why" the constants $C_{p}$ and $E_{p}$ are so ubiquitous in the $L^{p}$ theory of singular integrals endowed with a great degree of symmetry. They use Burkholder's method of differential subordination of martingales, which is at the root of many sharp inequalities for martingales $[\mathrm{Bu}]$. An important application of these probabilistic techniques, to be found in [BW], are some good $L^{p}$ estimates for the Beurling-Ahlfors transform. See also [IM] for an approach through transference and Lindeman's article [Lin] for related results in higher dimension.

Observe that, in view of Remark 1 in $\S 1$, estimates (2.1) and (2.2) hold with $R^{c}$ and $R^{b}$ instead of $Q^{c}$ and $Q^{b}$ respectively, with the same constants.

One can show that the $L^{p}$ operator norms of $R_{s}$ and $R_{t}$ are dimension free. More precisely, we have the following

Proposition 3. [Li] The $L^{p}$ operator norm of $R_{s}$ (thus also $\left.R_{t}\right)$ is bounded above by

$$
\frac{\pi^{1 / 2}}{2 \sqrt{2}}\left(\frac{p}{p-1}\right)^{1 / 2} B_{p},
$$

where $B_{p}=C_{M, p}+C_{p}$ and $C_{M, p}$ is the $L^{p}$ norm of the maximal truncated Hilbert transform on $\mathbb{S}^{1}$.

The proof makes use of a transference argument from the circle group to $S O(n)$.

It would be interesting to know more about the $L^{p}$ norms of single components of $R^{c}$ and $R^{b}$. More explicitly, the imbedding of $\mathbb{S}^{n-1}$ in $\mathbb{R}^{n}$ identifies $X=\nabla_{\mathbb{S}^{n-1}}$ with a vector in $\mathbb{R}^{n}: X=\left(X_{1}, \ldots, X_{n}\right)$. It is then reasonable to call $X_{j} \circ O$ the $j^{\text {th }}$ component of $R^{c}\left(\right.$ resp. $\left.R^{b}\right)$, where $O=\left(-\Delta_{\mathbb{S}^{n-1}}\right)^{-\frac{1}{2}}\left(\right.$ resp. $\left.O=\left(\frac{\partial}{\partial r}\right)^{-1}\right)$.

\section{3. $\mathbf{S O}(\mathbf{n}), \mathbb{S}^{n-1}$ and $Q^{c}$}

A notion of Riesz transform can be defined on Lie groups. The analysis of these transforms is easier if the Lie group $G$ is compact, the case that we are going to discuss in this section, following [St1 Chapt.I, II]. 
Let $G$ be a compact Lie group of dimension $m$ and let $\mathcal{G}$ be its Lie algebra, the linear space of all left invariant vector fields $X$ on $G$. $\mathcal{G}$ can be identified with the tangent space $T_{e} G$ to $G$ at $e$, the identity of $G$.

There exists a differential operator $\Delta_{G}$ such that

(i) $\Delta_{G}$ is the Laplace-Beltrami operator for a biinvariant metric on $G$;

(ii) $\Delta_{G}=\sum_{j=1}^{m} a_{j, k} X_{j} X_{j}$, where $\left\{X_{1}, \ldots X_{m}\right\}$ is an orthonormal basis for $\mathcal{G}$ with respect to the metric in (i).

For $j=1, \ldots, m$, we can formally define a Riesz transform $P_{j}=X_{j} \circ$ $\left(-\Delta_{G}\right)^{-\frac{1}{2}}$. In order to see that this definition agrees with the general "cylindrical" definition given in $\S 0$, for $a \in G$ let $\operatorname{Pf}(a)=\sum_{j=1}^{m} P_{j} f(a) X_{j}(a)$, which is a vector in $T_{a} G$, easily identified as $P f=\nabla_{G} \circ\left(-\Delta_{G}\right)^{-\frac{1}{2}} f$.

E. M. Stein proved that, for $1<p<\infty, P$ is bounded from $L^{p}(G)$ to itself and that the constant $c_{p}$ in the inequality $\left\|P_{j} f\right\|_{p} \leq c_{p}\|f\|_{p}$ can be chosen to be independent of the particular compact Lie group $G$. An integration by parts shows that

Remark 3. $\|P f\|_{2}=\|f\|_{2}$.

We are interested in $G=S O(n)$, in view of its connections with $\mathbb{S}^{n-1}$. Consider $S O(n)$ as a group of $n \times n$ matrices. Then $S O(n) \hookrightarrow \mathbb{R}^{n^{2}}$ and this imbedding induces on $S O(n)$ a metric which is biinvariant under the action of $S O(n)$ on itself.

The Lie algebra $s o(n)$ of $S O(n)$ can be identified with the space of all $n \times n$ skew symmetric matrices. If we rescale the metric on $S O(n)$ by a factor of $\frac{\sqrt{2}}{2}$, we see that an orthonormal basis of $s o(n)$ is provided by $\left\{X_{l m}=\left[a_{j, k}^{l m}\right]_{j, k}\right.$ : $1 \leq l<m \leq n\}$, where the matrix $\left[a_{j, k}^{l m}\right]_{j, k}$ is defined by $a_{l, m}^{l m}=-1, a_{m, l}^{l m}=1$ and all other entries are 0 . The gradient of a function $F$ on $S O(n)$ is given by $\nabla_{S O(n)} F=\sum_{l<m} X_{l m}(F) X_{l m}$.

An integration by parts shows that $X_{l m}{ }^{*}=-X_{l m}$, where ()$^{*}$ is the adjoint. We then have $\Delta_{S O(n)}=\sum_{l<m} X_{l m} X_{l m}$, the Laplace-Beltrami operator associated with our metric and the Riesz transform $P_{l m}$ on $S O(n)$ is given by

$$
P_{l m}=X_{l m} \circ\left(-\Delta_{S O(n)}\right)^{-\frac{1}{2}} .
$$

In the special case of $S O(n)$ we can say something more precise about the $L^{p}$ size of the Riesz transform. Let $C_{p}, E_{p}$ and $p^{*}$ be the constants defined in $\S 2$ and let $L_{0}^{p}(S O(n))$ be the space of those $F \in L^{p}(S O(n))$ with null average on $S O(n)$.

Theorem 2. [A1] If $F \in L_{0}^{p}(S O(n))$ is a real valued function, then the following inequalities hold:

$$
\frac{1}{2\left(p^{*}-1\right)}\|F\|_{p} \leq\|P F\|_{p} \leq 2\left(p^{*}-1\right)\|F\|_{p}
$$




$$
\begin{gathered}
\left\|P_{l m} F\right\|_{p} \leq C_{p}\|F\|_{p} ; \\
\left\|\left[F^{2}+\left|P_{l m} F\right|^{2}\right]^{\frac{1}{2}}\right\|_{p} \leq E_{p}\|F\|_{p} .
\end{gathered}
$$

Moreover, the constants in (3.3) and (3.4) are best possible.

The proof of this theorem is, again, based on the probabilistic methods of $[\mathrm{BW}]$. We will omit the proof here (see [A1] for the proof); instead, we sketch now a proof that Theorem 2 implies (2.1), (2.3) and (2.5) in Theorem 1.

As we mentioned before, we can view $\mathbb{S}^{n-1}$ as the homogeneous space $S O(n) / S O(n-1)$, where $S O(n-1)$ is the Lie group of rotation that fix the north pole $e_{n}=(0, \ldots, 0,1) \in \mathbb{S}^{n-1}$. Let $\pi$ be the projection: $S O(n) \rightarrow \mathbb{S}^{n-1}$, defined by $\pi(a)=a e_{n}, a \in S O(n)$. Recall that the adjoint representation Ad of a Lie group $G$ associates to each $a \in G$ a linear map $\operatorname{Ad}(a): \mathcal{G} \rightarrow \mathcal{G}$ by $\operatorname{Ad}(a) X=\left.\frac{d}{d t}\right|_{t=0} a \exp (t X) a^{-1}$. Since the metric on $G$ is biinvariant, $\operatorname{Ad}(a)$ is an isometry for each $a \in G$.

Elementary arguments in Lie theory show that, if $f: \mathbb{S}^{n-1} \rightarrow \mathbb{R}$,

$$
\Delta_{S O(n)}(f \circ \pi)(a)=\left(\Delta_{\mathbb{S}^{n-1}} f\right)(\pi(a))
$$

and

$$
\left(T_{l m} f\right)(\pi(a))=\left(\operatorname{Ad}\left(a^{-1}\right) X_{l m}\right)(f \circ \pi)(a) .
$$

Hence, by definition of $Q_{l m}^{c}$,

$$
\left(Q_{l m}^{c} f\right)(\pi(a))=\left[\operatorname{Ad}\left(a^{-1}\right) X_{l m} \circ\left(-\Delta_{S O(n)}\right)^{-1 / 2}\right] f(\pi(a))
$$

which is a "twisted version" of $P$.

Using the fact that the Hausdorff measure on $\mathbb{S}^{n-1}$ is proportional to the push forward of the Haar measure on $S O(n)$, i.e., that there exists $\mu>0$ such that $\int_{S O(n)}(f \circ \pi)=\mu \int_{\mathbb{S}^{n-1}} f$, it is not too difficult to check that, for instance, (3.3) together with (3.7) implies (2.3). This argument, in the same way, shows that the sharpness of $C_{p}$ in (2.3) implies that $C_{p}$ is best possible in (3.3) as well.

\section{Spherical Riesz transforms and system of conjugate harmonic functions}

Consider a system of conjugate harmonic functions in unit ball $\mathbb{B}^{n}$ which are continuous on $\overline{\mathbb{B}}^{n}$, and then restrict the functions to $\mathbb{S}^{n-1}$. Namely, suppose that $u_{1}, \ldots, u_{n}$ are functions in $\mathbb{B}^{n}$ which satisfy

$$
\sum_{j=1}^{n} \frac{\partial u_{j}}{\partial x_{j}}=0, \quad \frac{\partial u_{j}}{\partial x_{k}}=\frac{\partial u_{k}}{\partial x_{j}}, \quad \text { for all } j \neq k, \quad j, k=1, \ldots, n
$$

in $\mathbb{B}^{n}$ and are such that $u_{1}(0)=\cdots=u_{n}(0)=0$. Let $f_{j}=\left.u_{j}\right|_{\mathbb{S}^{n-1}}$. Then $f_{1}, \ldots, f_{n}$ can be called a Riesz system on $\mathbb{S}^{n-1}$. Observe that none of the Riesz transforms we introduced in $\S 1$ leads to such a Riesz system. In fact, solutions 
of (4.1) are in a bijection with the space of harmonic functions $h$ in $\mathbb{B}^{n}$ such that $h(0)=0$, the correspondence being that which associates to $h$ the $n$-tuple $u_{j}=\frac{\partial h}{\partial x_{j}}, j=1, \ldots, n$. The Riesz transform $R^{b}$ corresponds to the tangential part of $\nabla h$, while the Riesz system corresponds to $\nabla h$ itself. More specifically, if $f$ is a function defined on $\mathbb{S}^{n-1}$ and if $e_{j}=(0, \ldots, 0,1,0, \ldots, 0)$, where 1 appears in the $j^{\text {th }}$ coordinate, then

$$
f_{j}=\left(e_{j}, R^{b} f+\frac{x}{|x|} f\right)
$$

$j=1, \ldots, n$, is a Riesz system, $($,$) being the inner product in \mathbb{R}^{n}$.

Several $L^{p}$ inequalities tell us that $n-1$ components of a Riesz system control all others; for example,

$$
\left\|f_{n}\right\|_{L^{p}\left(\mathbb{S}^{n-1}\right)} \leq A_{p, n}\left\|\left[\sum_{j=1}^{n-1}\left|f_{j}\right|^{2}\right]^{\frac{1}{2}}\right\|_{L^{p}\left(\mathbb{S}^{n-1}\right)},
$$

and

$$
\left\|f_{n}\right\|_{L^{p}\left(\mathbb{S}^{n-1}\right)} \leq B_{p} \sum_{j=1}^{n-1}\left\|f_{j}\right\|_{L^{p}(\mathbb{S} n-1)}
$$

where $1<p<\infty$.

Inequality (4.2) was first proved by Kuran $[\mathrm{Ku}]$. The fact that $A_{p, n}$ must grow with $n$ can be verified on $u_{j}=\frac{\partial h_{0}}{\partial x_{j}}$, where $h_{0}(x)=-\frac{1}{2} \sum_{j \neq n} x_{j}^{2}+\frac{(n-1)}{2} x_{n}^{2}$. This gives a value $A_{p, n} \geq K_{p} \sqrt{n}$, where $K_{p}$ is a constant that only depends on $p$. Essén [Es] obtained that, for $1<p \leq 2, A_{p, n} \leq\left(\frac{n+1}{p-1}\right)^{\frac{1}{p}}$. This estimate can be improved. The first author proved that $A_{p, n} \leq\left(p^{*}-1\right) \sqrt{n-1}, 1<p<\infty$. See $[A 2]$.

Bennett [Be] used a probabilistic Littlewood-Paley theory to prove that for all $p>1$ (4.3) holds with a constant $B_{p}$, which does not depend on the dimension. Bennett also shows that in an inequality similar to (4.3),

$$
\left\|f_{n}\right\|_{L^{p}\left(\mathbb{S}^{n-1}\right)} \leq D_{p, n}\left(\sum_{j=1}^{n-1}\left\|f_{j}\right\|_{L^{p}\left(\mathbb{S}^{n-1}\right)}^{p}\right)^{\frac{1}{p}}
$$

the constant $D_{p, n}$ will tend to infinity with $n$ at least as fast as $n^{\frac{1}{p}}$, for all $p>1$. His example is, again, $h_{0}$.

In order to compare estimates (4.2)-(4.4) it is useful to know the following inequalities

$$
B_{p} \leq D_{p, n} \leq A_{p, n} \text {, if } 1<p \leq 2 \text { and } B_{p} \leq A_{p, n} \leq D_{p, n}, \text { if } 2 \leq p<\infty .
$$


Moreover, $A_{p, n} \leq \sqrt{n} B_{p}$ if $1<p \leq 2$. For a proof, it suffices to compare the right hand sides of (4.2)-(4.4) by making use of Minkowsky's and Hölder's inequalities.

In order to control the $n^{\text {th }}$ component of a Riesz system we need all other $n-1$ components. Examples are provided by Riesz systems of the form $f_{1}=$ $\frac{\partial h}{\partial x_{1}}, \ldots, f_{n}=\frac{\partial h}{\partial x_{n}}$, where $h$ is a harmonic function that only depends on $x_{1}, x_{2}$.

Estimate (4.2) has a weak $L^{1}$ counterpart. Let $\sigma$ be the natural Hausdorff measure on $\mathbb{S}^{n-1}$.

Theorem 3. [A2] Let $f_{1}, \ldots, f_{n}$ be a Riesz system on $\mathbb{S}^{n-1}$. Then for $\lambda>0$,

$$
\lambda \sigma\left(\left\{\xi \in \mathbb{S}^{n-1}:\left|f_{n}(\xi)\right|>\lambda\right\}\right) \leq 4 \sqrt{n}\left\|\left(\sum_{j=2}^{n}\left|f_{j}\right|^{2}\right)^{\frac{1}{2}}\right\|_{1} .
$$

Furthermore, there exists a universal constant $C>0$ such that, for any $n$ there exist $\lambda>0$ and a Riesz system $f_{1}, \ldots, f_{n}$ such that

$$
\lambda \sigma\left(\left\{\xi \in \mathbb{S}^{n-1}:\left|f_{n}(\xi)\right|>\lambda\right\}\right) \geq C \sqrt{n}\left\|\left(\sum_{j=2}^{n}\left|f_{j}\right|^{2}\right)^{\frac{1}{2}}\right\|_{1}
$$

It would be interesting to have similar weak $L^{1}$ analogues of (4.3) and (4.4).

It seems that there is only scant connection between estimates (4.2)-(4.4) and the ones stated in $\S 1$ for $Q^{c}, Q^{b}, R_{s}$ and $R_{t}$.

\section{Acknowledgements}

The authors are indebted to Professor Albert Baernstein and Professor Guido Weiss for valuable advice and many useful discussions. They also thank the referee for directing their attention to some references.

\section{References}

[A1] N. Arcozzi, Riesz transforms on compact Lie groups, spheres and Gauss space, preprint, 1997.

$[\mathrm{A} 2] \_$Strong $(p, p)$ and weak $(1,1)$ inequalities for systems of conjugate harmonic functions in Euclidean domains, preprint, 1997.

[Bak] D. Bakry, Etude probabiliste des transformées de Riesz et de l' espace $H^{1}$ sur les sphéres, Sém. de Prob. XVIII, 197-218, Lecture Notes in Math., 1059, Springer, 1984.

[Bañ] R. Bañuelos, Martingale transforms and related singular integrals, Trans. Amer. Math. Soc. 293 (1986), 547-563.

[BW] R. Bañuelos and G. Wang, Sharp inequalities for martingales with applications to the Beurling-Ahlfors and Riesz transforms, Duke Math. J., 80 (1995), 575-600.

[Be] A. Bennett, Probabilitistic square functions and a priori estimates, Trans. Amer. Math. Soc. 291, (1985), 159-166.

[Bu] D. L. Burkholder, Sharp inequalities for martingales and stochastic integrals, Astérisque 157-158 (1988), 75-94. 
[CW1] R. Coifman and G. Weiss, Invariant systems of conjugate harmonic functions associated with compact Lie groups, Studia Math. 44 (1972), 301-308.

[CW2] _ Transference methods in analysis, CBMS Regional Conf. Ser. in Math. 31, 1977.

[DF] J. Duoandikoetxea and J. L. Rubio de Francia, Estimations indépendantes de la dimension pour les transformées de Riesz, C. R. Acad. Sci. Paris Sér. I Math. 300 (1985), 193-196.

[Es] M. Essén, A superharmonic proof of the M. Riesz conjugate function theorem, Ark. Math. 22 (1984), 241-249.

[IM] T. Iwaniec and G. Martin, Riesz transforms and related singular integrals, J. Reine Angew. Math., 473 (1996), 25-57.

[Ku] Ü. Kuran, $n$-Dimensional extensions of theorems on conjugate functions, Proc. London Math. Soc. (3) 15 (1965), 713-730.

[KV1] A. Korányi and S. Vági, Singular integrals in homogeneous spaces and some problems of classical analysis, Ann. Scuola Norm. Sup. Pisa (3) 25 (1971), 575-648.

[KV2] A. Korányi and S. Vági, Group theoretic remarks on Riesz systems on balls, Proc. Amer. Math. Soc. 85 (1982), 200-205.

[Le] D. Levine, Systems of singular integral operators on spheres, Trans. Amer. Math. Soc. 144 (1969), 493-522.

[Li] X. Li, Thesis, Washington University, 1996.

[Lin] A. Lindeman II, Martingales and the $n$-dimensional Beurling-Ahlfors transform, preprint, 1995.

[Me] P. Meyer, Démonstrations probabilistes des inégalités de Littlewood-Paley, Sém. de Prob. X, Lecture Notes in Math., 511, Springer, 1976.

[Pic] S. K. Pichorides, On the best values of the constants in the theorems of M. Riesz, Zygmund and Kolmogorov, Studia Math. 44 (1972), 165-179.

[Pis] G. Pisier, Riesz transforms: A simple Analytic proof of P.A.Meyer's Inequality, Sém. de Prob. XXII, Lecture Notes in Math., 1321, Springer, 1988.

[RW] F. Ricci and G. Weiss, A characterization of $H^{1}\left(\Sigma_{n-1}\right)$, Proc. Sympos. Pure Math., XXXV, 1 (1979), 289-294.

[St1] E. M. Stein, Topics in harmonic analysis related to the Littlewood-Paley theory, Ann. of Math. Stud., 63, Princeton Univ. Press, Princeton, N.J., 1970.

[St2] S Singular integrals and differentiability properties of functions, Princeton Math. Ser., 30, Princeton University Press, Princeton, N.J., 1970.

[St3] Soc. 9 (1983), 71-73.

[St4] - Three variations on the theme of maximal functions, Recent Progress in Fourier Analysis, 229-244, I. Peral and J.-L. Rubio de Francia (Editors), NorthHolland Math. Stud., 111, North-Holland 1985.

[SW] E. M. Stein and G. Weiss, Introduction to Fourier analysis on Euclidean spaces, Princeton Math. Ser., 32, Princeton University Press, Princeton, N.J., 1971.

Via Monte Cengio 11, 20138 Milano, ItAly

E-mail address: arcozzi@mat.unimi.it

Department of Mathematics, Washington University, Campus Box 1146, St. Louis, MO 63130-4899, USA

E-mail address: xli@math.wustl.edu 